\title{
Quantum Transport and Thermoelectric Properties of InAs/GaSb Superlattices
}

\author{
J.-F. LIN and D. Z.-Y. TING* \\ Department of Physics, National Tsing Hua University, Hsinchu, Taiwan 300, ROC
}

\begin{abstract}
In recent years, artificially layered microstructure have been considered as candidates for better thermoelectrics. In this work we examine transport properties of the type-II broken-gap In $\mathrm{As} / \mathrm{GaSb}$ superlattice. We use the effective bond orbital model for an accurate description of the band structures. Theoretical results of thermoelectric transport coefficients and the dimensionless figure of merit for an $(8,8)$-InAs/GaSb type-II superlattice are presented.
\end{abstract}

Keywords: Thermoelectric, type-II superlattice, superlattice transport, InAs, GaSb

\section{INTRODUCTION}

From about 1940 to 1965 , many scientists made tremendous effort looking for superior thermoelectric materials. The advantage of using these solid state devices are compactness, quietness (no moving parts), freedom from corrosion, localized heating or cooling, and reliability. The effort dwindled eventually because performance of most thermoelectric materials found at that time were too poor to be used for practical commercial applications. Recently, there is a renewed interest in this area $[1,2]$, driven by the following reasons: First, thermoelectric technology is environmentally cleaner than traditional compressor-based refrigeration technology since it does not use chlorofluorocarbons. Second, several new materi- als were identified as potential candidates for better thermoelectrics, including the filled skutterudite antimonides [3] and $\mathrm{PbTe} / \mathrm{Pb}_{1-x} \mathrm{Eu}_{x} \mathrm{Te}$ multiple-quantum-well structures [4]. Among the new materials, superlattices attracted many scientists' attention $[5,6,7,8,9]$. The interest can be traced back to the quantitative results first obtained by Hicks and Dresselhaus [5] where huge enhancement of thermoelectric properties was predicted for superlattice structures.

In the present work, we focus our interest on the type-II broken-gap InAs/GaSb superlattices. Superlattices consisting of combinations of III$\mathrm{V}$ semiconductors with type-II band alignments are of interest for infrared applications, including IR detectors [10] and laser diodes [11, 12]. This is because their energy gaps can be made smaller

\footnotetext{
* Corresponding author.
} 
than their constituents. However, for most of these applications, cooled operation are desirable for good performance. If reasonable thermoelectric properties of such superlattice structure could be obtained, directly integration of thermoelectric cooling devices with a set of IR detectors or laser diodes may offer some advantage. Our aim here is to present the early results of our ongoing theoretical effort to identify and characterize the thermoelectric properties of the type-II brokengap InAs/GaSb superlattices. In Sec. II, the theoretical framework for calculating electronic contributions to the thermoelectric properties of superlattices using realistic band structure models is presented. Our results for an $(8,8)-\operatorname{InAs} / \mathrm{GaSb}$ superlattice is presented in Sec. III.

\section{MODEL}

The dimensionless figure of merit for a thermoelectric material is given by $[14,15]$

$$
Z T=\frac{S^{2} \sigma T}{\kappa}
$$

where $S$ is the thermopower (thermoelectric power or Seebeck coefficient), $\sigma$ is the electrical conductivity, $\kappa$ is the total thermal conductivity $\left(\kappa=\kappa_{1}+\kappa_{\mathrm{e}}\right.$, the lattice and electronic contributions, respectively), and $T$ is the temperature. The maximum Coefficient Of Performance $(C O P)$ is directly related to the dimensionless figure of merit of the material by $[14,15]$

$$
C O P=\frac{T_{\mathrm{c}}}{T_{\mathrm{h}}-T_{\mathrm{c}}} \frac{\sqrt{1+Z T}-T_{\mathrm{h}} / T_{\mathrm{c}}}{\sqrt{1+Z T}+1},
$$

where $T_{\mathrm{c}}, T_{\mathrm{h}}$ are the temperatures at the cold and hot junctions, respectively, and $T$, which is equal to $\left(T_{\mathrm{h}}+T_{\mathrm{c}}\right) / 2$, is the mean absolute temperature, for a single-stage thermoelectric cooler. As $Z T$ goes to infinity, the COP goes to the thermodynamic limit of Carnot efficiency.
The low-field transport coefficients for thermoelectric materials are defined by

$$
\begin{gathered}
\mathbf{J}=\sigma \mathbf{E}-\sigma S \nabla T \\
\mathbf{J}_{\mathbf{Q}}=T \sigma S \mathbf{E}-\kappa_{0} \nabla T
\end{gathered}
$$

where $\mathbf{J}$ is the electric current density, $\mathbf{J}_{\mathbf{Q}}$ the thermal current density, $\mathbf{E}$ the electric field, $T$ the temperature, $\sigma$ the electrical conductivity, $S$ the thermopower, and $\kappa_{0}$ the thermal conductivity at zero electric field. In general, $\sigma, S$, and $\kappa_{0}$ are $3 \times 3$ matrices in the Cartesian coordinates. Note that the value of $\kappa$ needed for computing $Z$ is the total heat flow for zero electrical current, so it is given by

$$
\kappa=\kappa_{0}-T \sigma S^{2}+\kappa_{l}
$$

where $\kappa_{l}$ is the lattice contribution to the thermal conductivity.

Using the relaxation time approximation to the Boltzmann equation, the transport coefficients are given by [16]

$$
\begin{gathered}
\sigma=e^{2} \int_{-\infty}^{\infty} d \varepsilon\left(-\frac{\partial f_{0}}{\partial \varepsilon}\right) \mathbf{\Sigma}(\varepsilon) \\
T \sigma S=-e \int_{-\infty}^{\infty} d \varepsilon\left(-\frac{\partial f_{0}}{\partial \varepsilon}\right) \mathbf{\Sigma}(\varepsilon)(\varepsilon-\mu) \\
T \kappa_{0}=\int_{-\infty}^{\infty} d \varepsilon\left(-\frac{\partial f_{0}}{\partial \varepsilon}\right) \mathbf{\Sigma}(\varepsilon)(\varepsilon-\mu)^{2}
\end{gathered}
$$

where $\mu$ is the chemical potential, $e$ the electron charge, and $f_{0}$ the Fermi-Dirac distribution function. Here $\mathbf{\Sigma}(\varepsilon)$ is given by:

$$
\mathbf{\Sigma}(\varepsilon)=\tau(\varepsilon) \sum_{k} \mathbf{v}(k) \mathbf{v}(k) \delta(\varepsilon-\varepsilon(k))
$$

where $\mathbf{v}(k)=\nabla_{k} \varepsilon(k) / \hbar$ is the semi-classical carrier velocity, $\varepsilon(k)$ is the dispersion relation for the carriers, and $\tau(\varepsilon)$ is the energy-dependent relaxation time tensor, taken to be $k$-independent. The summation is over the first Brillouin zone. Equations (6), (7), and (8) are the basic results of the theory of electronic contributions to the 
thermoelectric effects. It should be noted that phonon contributions are ignored here.

The theoretical scheme we used for calculating of the electronic structure of InAs/GaSb superlattices is the Effective Bond-Orbital Model (EBOM) [13]. This method combines the virtues of the $\mathbf{k} \cdot \mathbf{p}$ and the tight-binding methods. The basic idea is to use a minimum number of bond orbitals to describe, as accurately as possible, the most relevant portion of the bulk band structures, and then use them in a supercell calculation to obtain superlattice band structures. In our case, eight bond orbitals per unit cell, including the $s$ like bond orbitals with spin up and spin down, four bond orbitals each with total angular momentum $J=3 / 2$ (made of $p$-like states coupled with spin) and two additional bond orbitals each with total angular momentum $J=1 / 2$ (also made of $p$-like states coupled with spin), are used. This is because the superlattice states of interest contain admixture of both valence-band and conductionband characteristics. We assume that all the bond orbitals are sufficiently localized so that the interaction between orbitals separated farther than the nearest neighbor distance can be ignored. All nonvanishing interaction parameters can then be directly related to the effective masses or other band parameters of the $\mathbf{k} \cdot \mathbf{p}$ perturbation theory. Based on this model, an accurate band structure could be obtained for values of $\mathbf{k}$ near the zone center.

Our strategy for computing thermoelectric properties is as follows. First, we calculate the superlattice band structures by using SOBO model. Then, we perform full Brillouin zone integration to obtain $\boldsymbol{\Sigma}(\varepsilon)$. Finally, we use Equations (6), (7) and (8) to compute the transport coefficients.

\section{RESULTS AND DISCUSSION}

Figure 1 shows our calculated band structure for an InAs/GaSb superlattices eight monolayers of InAs and eight monolayeres of GaSb per period.

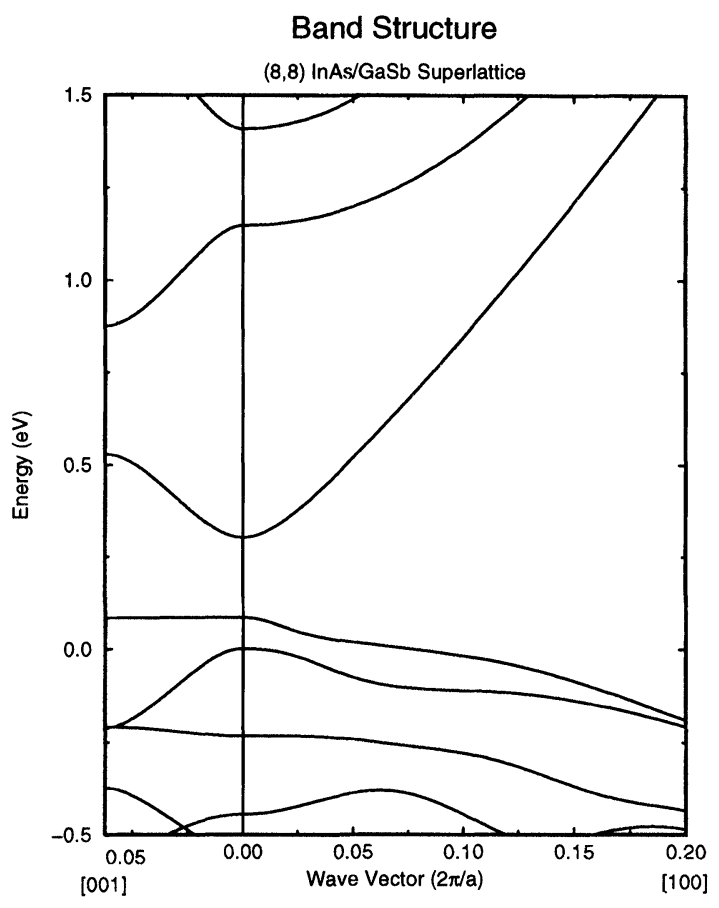

FIGURE 1 Illustration of band structure of $(4,4) \mathrm{InAs} / \mathrm{GaSb}$ superlattices. Note that in our calculation the strain effect is included.

The conduction band minimum of bulk InAs is taken to be at $0 \mathrm{eV}$. Experimental band offset value [17] between InAs and $\mathrm{GaSb}$ then puts the valence band top of $\mathrm{GaSb}$ at approximately 200 $\mathrm{meV}$ above the conduction band minimum of the InAs conduction band edge. In the figure, superlattice band structure perpendicular and parallel to growth directions are shown. The valence subband maximum is found at $0.086 \mathrm{eV}$, while the conduction subband minimum at $0.303 \mathrm{eV}$, yielding a superlattice bandgap of $0.217 \mathrm{eV}$. Note that the conduction subband structure along the superlattice growth direction is still very dispersive. This is due the broken-gap band alignment, the small InAs conduction band effective mass, and the relatively short period.

To compute thermal electric properties, we need to know relaxation times. Lacking specific knowledge of relaxation times in superlattice structures, we made an estimate from the mobility data for bulk InAs and $\mathrm{GaSb}$, and assume a value of 
$\tau(\varepsilon) \approx 10^{-13}$ sec. All of our calculations assume a temperature of $300 \mathrm{~K}$. In Figure 2, we plot the thermoelectric transport coefficients as functions of the chemical potential. Due to the fact that transport along the growth (transverse) direction is impeded by scattering from the superlattice interfaces, the transverse components $(z z)$ of electrical conductivity $(\sigma)$ and electronic contribution to thermal conductivity $\left(\kappa_{\mathrm{e}}\right)$ are always smaller than the parallel $(x x)$ components. The transverse components of all three transport coefficients show strong oscillations as functions of chemical potential. This can be understood by examining the superlattice minibands along the transverse ([001]) direction. We can easily see that the oscillations track the miniband edges.

In order to calculate the thermoelectric figure of merit for this structure we need the lattice contribution to the thermal conductivity. From the experimental data of bulk InAs and $\mathrm{GaSb}$, we take the lattice thermal conductivity of superlattice as the average of two bulk values, which is approximately $35 \mathrm{~W} / \mathrm{K} \mathrm{m}$. This procedure probably overestimates the values of lattice conductiv-

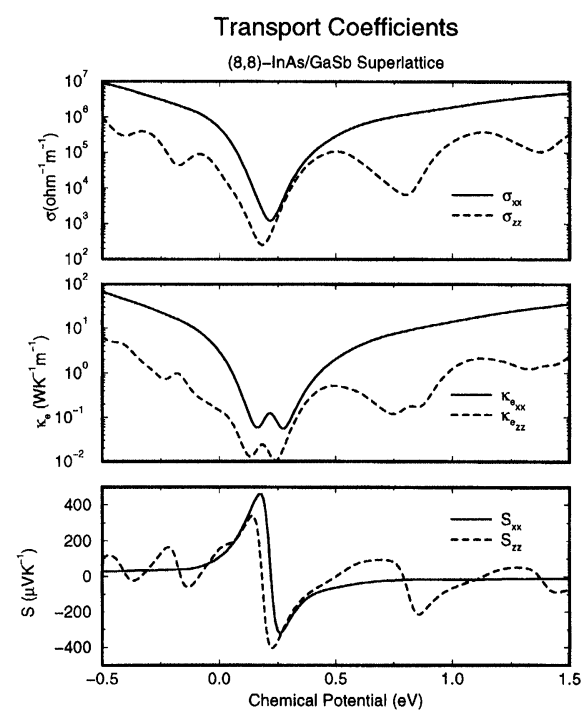

FIGURE 2 Shown are the conductivity, electronic contribution to the thermal conductivity, and thermopower vs. the chemical potential at $T=300 \mathrm{~K}$. ity since we would expect interface scattering to lower thermal conductivities. In Figure 3, we show the dimensionless figure of merit $Z T$ vs. the chemical potential. For parallel transport, the optimum value occurs when chemical potential is about $0 \mathrm{eV}$. Its value is about 0.033 which is not better than the bulk value. This result is opposite to that of Hicks' calculation [5] where $Z T$ for a type-I superlattices better than bulk materials. The difference is probably due to the much stronger inter-well coupling in the type-II broken gap superlattices.

While our calculations have carefully treated band structure effects, the models we used to estimate relaxation times and lattice thermal conductivities have been rather crude. In particular, it is likely that we considerably over-estimated the superlattice thermal conductivity [2]. These issues must be addressed before we could obtain a more realistic description of superlattice thermoelectric properties. In summary, we have calculated the thermoelectric properties, including conductivity, electronic contribution to thermal conductivity, and thermal power, for a type-II

Figure of Merit

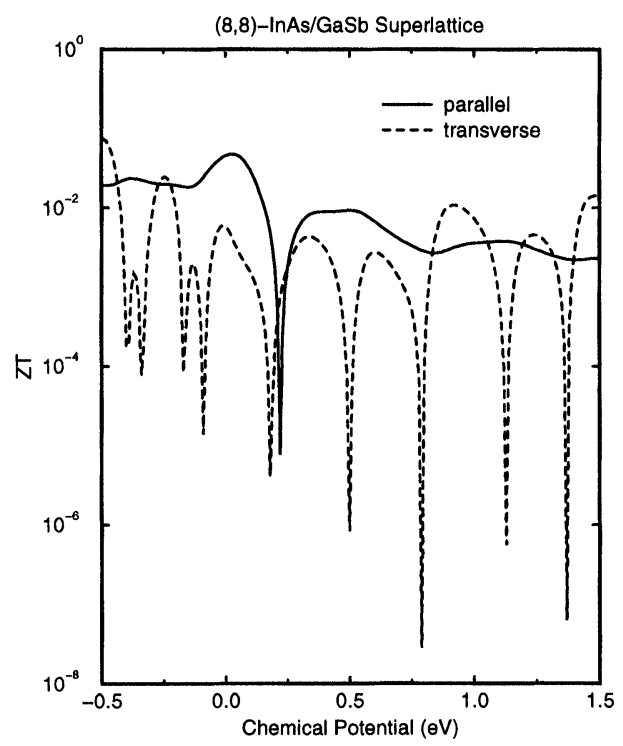

FIGURE $3 Z T$ vs. chemical potential for the $(8,8)-\operatorname{InAs} /$ $\mathrm{GaSb}$ superlattices. 
broken-gap InAs/GaSb superlattice using realistic band structure models. The computational tools developed form a basis for further explorations of superlattice thermoelectric properties.

\section{Acknowledgements}

The authors would like to thank C.C. Chi for helpful discussions. This work was supported by the ROC National Science Council under Grant No. NSC 86-2112-M-007-001. The use of ROC National Center for High-Performance Computing facilities is acknowledged.

\section{References}

[1] Tritt, T. M. (1996). "Thermoelectrics run hot and cold", Science, 272, 1276-1277.

[2] Mahan, G., Sales, B. and Sharp, J. "Thermoelectric materials: new approaches to an old problem", Physics Today, March 1997, 42-47.

[3] Sales, B. C., Mandrus, D. and Williams, R. K. (1996). "Filled skutterudite antimonides: a new class of thermoelectric materials", Science, 272, 1325-1328.

[4] Hicks, L. D., Harman, T. C., Sun, X. and Dresselhaus, M. S. (1996). "Experimental study of the effect of quantumwell structures on the thermoelectric figure of merit", Phys. Rev., B53, 10493-10496.

[5] Hicks, L. D. and Dresselhaus, M. S. (1993). "Effect of quantum-well structures on the thermoelectric figure of merit", Phys. Rev., B47, 12727-12731.

[6] Sofo, J. O. and Mahan, G. D. (1994). "Thermoelectric figure of merit of superlattices", Appl. Phys. Lett., 65(21), $2690-2692$.

[7] Mahan, G. D. and Lyon, H. B. (1994). "Thermoelectric devices using semiconductor quantum wells", J. Appl. Phys., 76(3), 1899-1901.

[8] Whitlow, L. W. and Hirano, T. (1995). "Superlattice applications to thermoelectricity", J. Appl. Phys., 78(9), $5460-5466$.

[9] Lin-Chung, P. J. and Reinecke, T. L. (1995). "Thermoelectric figure of merit of composite superlattices systems", Phys. Rev., B51, 13244-13248.
[10] Chow, D. H., Miles, R. H., Schulman, J. N., Collins, D. A. and Mcgill, T. C. "Type-II superlattices for infrared detectors and devices", Semi. Sci. Tech., v6(12C), 47-51 (1991 Dec.).

[11] Chow, D. H., Miles, R. H., Hasenberg, T. C., Kost, A. R., Zhang, Y. H., Dunlap, H. L. and West, L. "Mid-wave infrared diode-lasers based on $\mathrm{GaInSb/InAs} \mathrm{and} \mathrm{InAs/}$ AlSb superlattices", Appl. Phys. Let., 67 (25), 3700-3702 (1995 Dec. 18).

[12] Miles, R. H., Chow, D. H., Zhang, Y. H., Brewer, P. D. and Wilson, R. G. "Midwave infrared stimulated-emission from a GaInSb/InAs superlattices", Appl. Phys. Let., 66(15), 1921 -1923 (1995 April. 10).

[13] Chang, Y.-C. (1988). "Bond-orbital models for superlattices", Phys. Rev., B37, 8215-8222.

[14] Ioffe, A. F. Semiconductor Thermoelements and Thermoelectric Cooling (Infosearch Ltd., 1957).

[15] Goldsmid, H. J. Applications of Thermoelectricity (London: Methuen \& Co Ltd. New York: John Wiley \& Sons Inc., 1960).

[16] Ashcroft, N. W. and Mermin, N. D. Solid State Physics, Chapter 13 (Harcourt Brace College Publishers, International Edition).

[17] Sai-Halasz, G. A., Chang, L. L., Walter, J. M., Chang, C. A. and Esaki, L. (1978). Solid State. Commun., 27, 935.

\section{Authors' Biographies}

Jie-Feng Lin received a B.S. degree in Electrophysics from the National Chiao Tung University, Taiwan in 1995, and an M.S. Degree in Physics from the National Tsing Hua University, Taiwan in 1997. His involves theoretical studies of semiconductor heterostructures.

David Z.-Y. Ting is an Associate Professor of Physics at the National Tsing Hua University in Hsinchu, Taiwan, ROC, and a Visiting Research Associate at the California Institute of Technology. His research activities include theoretical studies of electronic and optical properties of semiconductor alloys and heterostructures, quantum transport in nanostructures and tunnel devices, and optical simulations. 

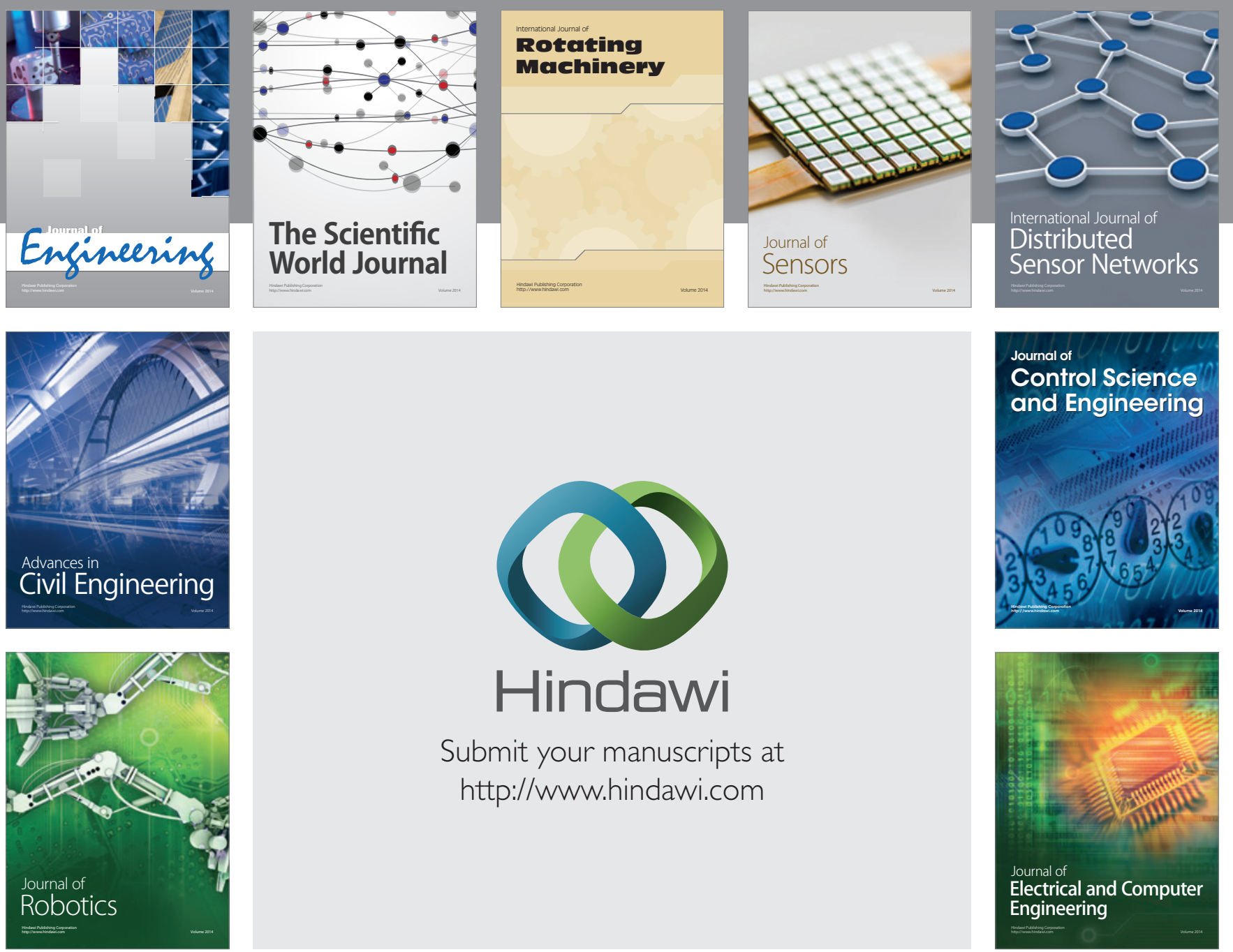

Submit your manuscripts at

http://www.hindawi.com
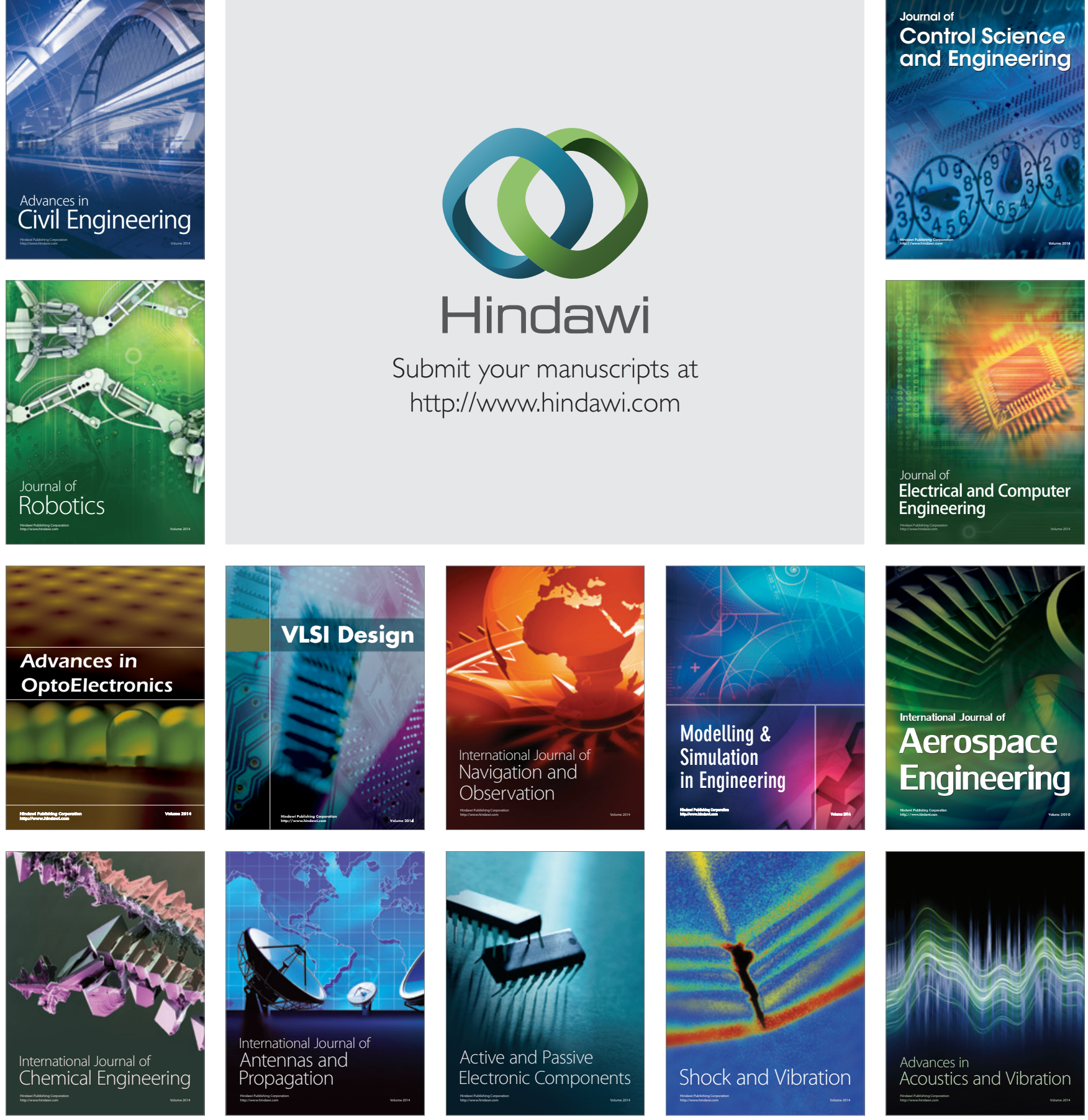